\title{
UNA FACULTAD UNA HISTORIA: ANALISIS ACADEMICO-SOCIO-POLÍTICO DE LA FORMACIÓN DE PROFESIONALES EN ENFERMERÍA. UNIVERSIDAD NACIONAL SAN LUIS GONZAGA DE ICA. PERÚ .1981-2012.
}

A Faculty one a Story:Academic analysis - socio-political training of professional nursing. National University of San Luis Gonzaga Ica. Perú .1981-2012.

Margarita Córdova Delgado ${ }^{1, a, d}$ Susana Alvarado Alfaro ${ }^{2, a, b}$ Heddy Manrique Manrique ${ }^{2, a, c}$ Paulo Tomaylla Palomino ${ }^{3}$; Nayarit Ortega Palomino ${ }^{3}$; Stefany Aguirre Alvarado ${ }^{3}$ Herhuay Mucha Mónica $^{3}$

${ }^{1}$ Facultad de enfermería de la Universidad San Luis Gonzaga de Ica. Ica, Perú. ${ }^{2}$ Hospital Regional de Ica, Perú; ${ }^{3}$ Estudiante de enfermería.

${ }^{a}$ Licenciada en enfermería, ${ }^{b}$ Magister en Educación con mención en Administración y Planificación de la Educación Superior. ${ }^{c}$ Maestría en Salud Pública; ${ }^{\mathrm{d}}$ Doctorado en ciencias de la Salud.

\section{RESUMEN}

Objetivo General: Determinar la historia de la formación de los profesionales en enfermería: en el aspecto académico-socio-político de la facultad de enfermería. Universidad Nacional San Luis Gonzaga de Ica. Perú 1981-2012. Material y métodos: Investigación aplicada descriptiva de corte transversal, con un paradigma cuali-cuantitativo, la población y muestra fueron docentes y egresados de la Facultad. Resultados: La Facultad en sus 25 años de formación Académica en el aspecto demanda para la Entrada: El ingreso/captación de alumnos: Existe Alta demanda de ingreso 2009-I: (ratio: 11,53), 2009-II (8,6\%). Para el proceso: Cuenta con 58 docentes; 09 con grado de Doctor, 21 con grado de magister. El promedio de alumnos por asignatura (2009- II, 2010-I, 2010- II y 2011-I) fue 45 por aula. La relación Alumnos/docentes fue 9,34 (2010-II). El promedio de estudiante/profesor en prácticas clínica (2010-I y 2010-II) 5,5. El promedio ponderado fue 15,02 , ( $\pm 1,17)$. El currículo establecido a inicios del desempeño académico en el año 1981-1999 fue de tipo tradicional. El año 2000-2001 el currículo de la Comisión Reorganizadora (CORE). Desde el año 2002 hasta la actualidad se viene desarrollando el currículo del tipo constructivista. La modalidad de titulación en los últimos años es por sustentación de tesis. En el aspecto social: El promedio de edad de los alumnos fue 21 años $( \pm 3,38)$. El 84,6\% proceden del departamento de Ica, 5,4\% de Lima, 4,8\% de Ayacucho y 2,3\% de Huancavelica. El $90 \%$ son de sexo femenino, $76 \%$ estudiaron en institución educativa estatal. En el aspecto político, impera un órgano de gobierno tipo Consejo de Facultad. Conclusiones: Existen diferencias relevantes entre las preferencias de los diferentes tipos de modalidades de titulación, con un declive en la preferencia respecto a la suficiencia académica, actualización académica y proyecto de inversión, se observa además una estable preferencia por la complementación académica y un notable ascenso en la titulación por modalidad de tesis. En los últimos años son el $100 \%$ la obtención de título universitario por

Palabras Claves: Análisis Académico, Análisis Socio-Político, Profesional Enfermería.

\section{SUMMARY}

General Objective: To determine the history of the training of nurse practitioners: the socio-politicalacademic nursing faculty look. San Luis Gonzaga National University of Ica. Peru from 1981 to 2012. Methods: Descriptive cross-sectional applied research, with qualitative and quantitative paradigm, population and sample were teachers and graduates of the Faculty. Results: The College in its 25 years of academic training in the aspect Demand for Entry: Entry / attract students: High demand exists Income 2009-I: (ratio: 11,53), 2009-II (8,6\%). For the process: It has 58 teachers; Doctor grade 09, grade 21 magister. The average number of students per course (2009 - II, 2010-I, 2010 - 2011-I and II) was 45 per classroom. The Student / teacher ratio was 9,34 (2010-II). The average student / 
teacher ratio in clinical practice (2010-I and 2010-II) 5,5. The weighted average was $15,02( \pm 1,17)$. The curriculum established at the beginning of the academic performance in the year 1981-1999 was of the traditional type. The year 2000-2001 the curriculum Reorganizing Commission (CORE). Since 2002 to the present has been developing constructivist curriculum. The titration method in recent years is supporting the thesis. On the social side: The average age of students was 21 years $( \pm 3,38) .84,6 \%$ are from the department of Ica, Lima 5,4\%, 4,8\% and 2,3\% of Ayacucho to Huancavelica. 90\% were female, $76 \%$ studied in state educational institution. On the political side, there remains a body of Faculty Council type government. Recommendations: Continue with this type of research because it allows to know the history of the faculty and academic and political partner replied.

Keywords: Academic Review, Socio Political Analysis, Professional Nursing.

\section{INTRODUCCION}

El proceso de formación de los profesionales en la Educación Superior debe estar intencionalmente orientado a formar un ciudadano que reúna las condiciones que la sociedad actual demanda: sujetos altamente comprometidos con la historia y las tradiciones de su medio, profundamente reflexivos, capaces de comportarse, esencialmente humanos, con capacidad plena para el análisis, la argumentación, preparados para asumir los desempeños laborales que las distintas profesiones requieren; con todas las posibilidades para insertarse en el vertiginoso avance de la ciencia, la tecnología, el arte y dispuestos a crecer tanto en el orden de la preparación técnica y profesional como en sus condiciones personales y espirituales. Lo que se resume en profesionales comprometidos, flexibles y trascendentes.

La Facultad de Enfermería se apertura en el año 1981 como programa de enfermería adscrita a la Facultad de Medicina Humana. Su creación se debió a una necesidad imperiosa de que la Ciudad de Ica debía de contar con profesionales de enfermería que brinden atención integral al usuario, familia y grupos humanos en estado de salud o enfermedad, mediante las acciones de promoción, prevención, recuperación y rehabilitación de la salud de la población. El 31 de octubre del año 1984, el Consejo Universitario en pleno autoriza la creación de la Facultad de Enfermería, según Resolución Rectoral № 13315 designado como Decano interino al Dr. Luis Núñez Joseli, quien conduce la Facultad hasta el año 1986 y de esa fecha hasta el año 1989 el Dr. Manuel Alcibíades Ferrándiz Camacho. De allí en adelante el cargo del decanato recae en una docente enfermera de la facultad. Aunque la línea de discusión de este trabajo será la enfermería, consideramos oportuno dar a conocer, una serie de datos socios demográficos, con el fin de situarnos en el contexto que conforman sus características, y así poder comprender algunos de los aspectos que a continuación determinaremos (1).

La ciudad de Ica cuenta con una gran oferta educativa. La Universidad Nacional San Luis Gonzaga de Ica, es una institución de educación superior con personería jurídica de derecho público interno, creado por Ley №12495 del 20 de diciembre de 1955. Comenzó a funcionar por mandato del histórico cabildo abierto del pueblo de Ica del 18 de febrero de 1961 y oficialmente mediante RM № 137 del 18 de marzo del mismo año (2).

La Facultad de Enfermería se encuentra ubicada en la ciudad universitaria de la Universidad Nacional San Luís Gonzaga de Ica, Av. Los Maestros $s / n$ del distrito, provincia y departamento de Ica tiene en la actualidad 428 estudiantes matriculados y 58 docentes. Es una unidad fundamental de organización de formación académica y profesional integrada por profesores y estudiantes; funciona como órgano 
desconcentrado responsable de la formación profesional, investigación científica y tecnológica, la proyección social la producción de bienes y prestación de servicio (3).

El presente trabajo de investigación es de mucha importancia para el sector salud y educación porque permitirá conocer la historia de la Facultad de Enfermería de la Universidad Nacional San Luis Gonzaga de ICA. Desde 1981-hasta el .2012. El presente trabajo de investigación tiene por finalidad realizar un aporte práctico a la sociedad porque las conclusiones y recomendaciones que se obtendrán tendrán una aplicación cual es el de estar orgullosos de ser parte de esta Facultad, constituirse en la referencia inicial, que se vaya ampliando y estructurando hasta disponer de trabajos que puedan servir, tanto al investigador experimentado como al que inicia sus primeros pasos, de soporte adecuado para seguir o comenzar la investigación en el campo de la Historia de la Enfermería en Ica.

El objetivo del estudio fue determinar la historia de la formación de los profesionales en enfermería en el aspecto académico socio- político de la Facultad de Enfermería. Universidad Nacional San Luis Gonzaga de Ica.1981-2012.

\section{MATERIAL Y MÉTODOS}

La investigación fue aplicada descriptiva de corte transversal, con un paradigma cuali cuantitativo, de corte transversal. La población muestra estuvo constituida por docentes, autoridades y egresados. Las técnicas de recolección de datos son "un conjunto de pasos que se deben seguir durante el proceso de recolección de datos, para lo cual se diseña un formulario para registrar la información obtenida". En este caso, la técnica seleccionada de recolección de datos se realizó mediante un instrumento, el cual fue debidamente validado a través de consultas a juicio de expertos. Se utilizó como técnica la encuesta, cuestionarios, guías para historia de vida, autopsia verbal, guías de observación. Los datos fueron presentados en cuadros y gráficos, se utilizó la estadística descriptiva como la media aritmética y la desviación estándar. Las hipótesis se probó con la estadística inferencial, el analizador $Z$, se utilizó el software Excel y SPSS V. 15.0.

\section{RESULTADOS:}

La Facultad en sus 25 años de formación Académica en el aspecto demanda para la Entrada: El ingreso/captación de alumnos: Existe Alta demanda de ingreso 2009-I: (ratio: 11,53), 2009-II (8,6\%). Para el proceso: Cuenta con 58 docentes; 09 con grado de Doctor, 21 con grado de magister. El promedio de alumnos por asignatura (2009II, 2010-I, 2010- II y 2011-I) fue 45 por aula. La relación Alumnos/docentes fue 9,34 (2010II). El promedio de estudiante/profesor en prácticas clínica (2010-I y 2010-II) 5,5. El promedio ponderado fue $15,02,( \pm 1,17)$. El currículo establecido a inicios del desempeño académico en el año 1981-1999 fue de tipo tradicional. El año 2000-2001 el currículo de la Comisión Reorganizadora (CORE). Desde el año 2002 hasta la actualidad se viene desarrollando el currículo del tipo constructivista. La modalidad de titulación en los últimos años es por sustentación de tesis. En el aspecto social: El promedio de edad de los alumnos fue 21 años $( \pm 3,38)$. El $84,6 \%$ proceden del departamento de Ica, $5,4 \%$ de Lima, $4,8 \%$ de Ayacucho y $2,3 \%$ de Huancavelica. El $90 \%$ son de sexo femenino, $76 \%$ estudiaron en institución educativa estatal. En el aspecto político, impera un órgano de gobierno tipo Consejo de Facultad. 
TABLA 1

Número de Docentes según categoría. Facultad de Enfermería

\begin{tabular}{lrrrr}
\hline DOCENTE & DE & TC & TP & TOTAL \\
\hline PRINCIPAL & 2 & 8 & 0 & 10 \\
ASOCIADO & 11 & 16 & 0 & 27 \\
AUXILIAR & 2 & 2 & 17 & 21 \\
JEFE DE PRACTICA & 0 & 0 & 0 & 0 \\
TOTAL & 15 & 26 & 17 & 58 \\
\hline
\end{tabular}

TABLA 2

Creditaje Facultad de Enfermería 1981-2012

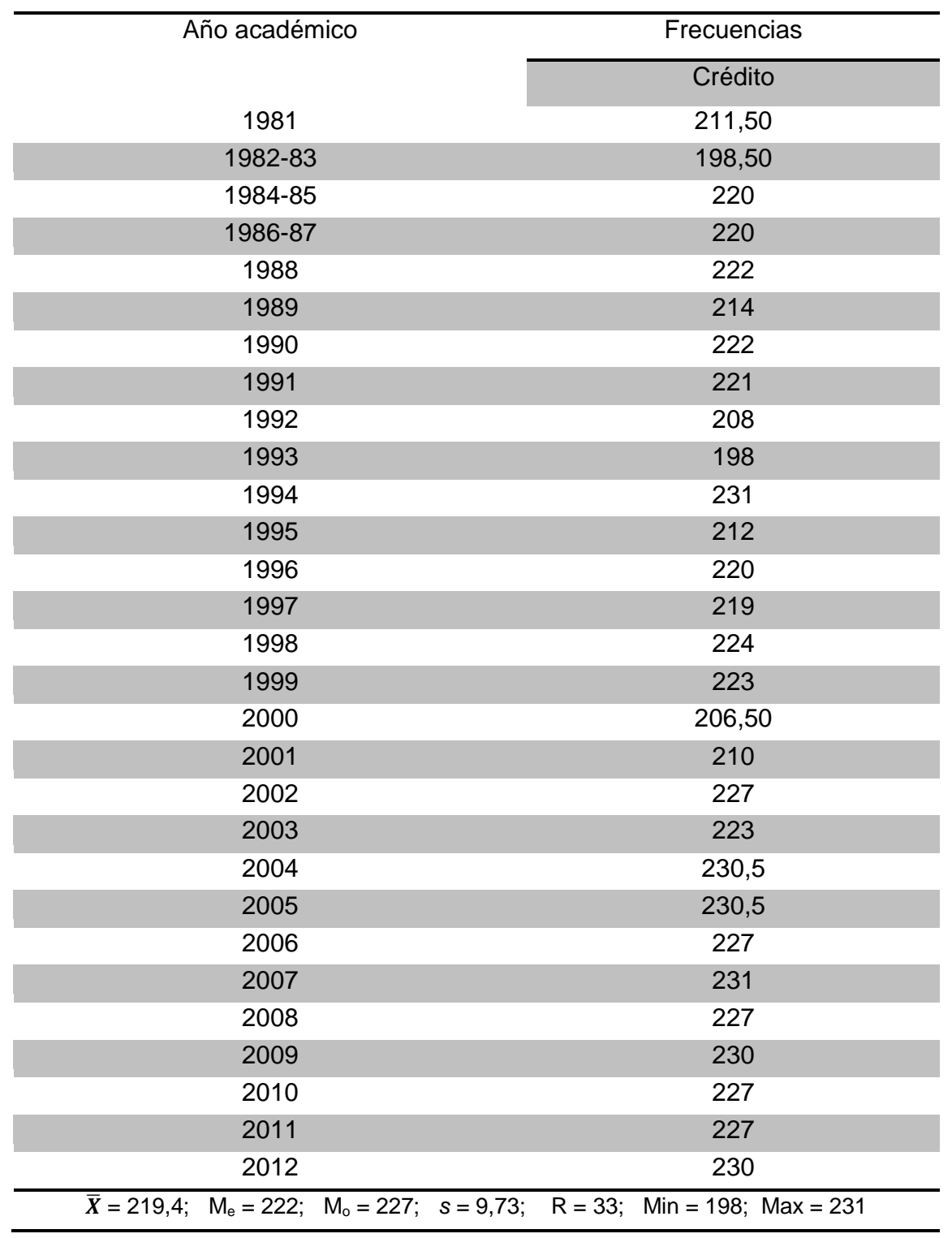




\section{TABLA 3}

Número de graduados por año. Facultad de Enfermería 1981-2012

\begin{tabular}{|c|c|c|c|}
\hline \multirow{3}{*}{ Año académico } & \multicolumn{3}{|c|}{ Frecuencias } \\
\hline & Absoluta & Porcentual & Acumulada \\
\hline & $\mathbf{f}_{\mathbf{i}}$ & $\mathbf{p}_{i} \%$ & $\mathbf{F}_{\mathbf{i}}$ \\
\hline 1981-1990 & 46 & 2,21 & 2,21 \\
\hline $1982-83-1991$ & 77 & 3,71 & 5,92 \\
\hline $1984-85-1994$ & 94 & 4,53 & 10,45 \\
\hline $1986-87-1995$ & 108 & 5,20 & 15,65 \\
\hline 1988-1996 & 53 & 2,55 & 18,20 \\
\hline 1989-1996 & 75 & 3,61 & 21,81 \\
\hline $1990-91-1997$ & 127 & 6,11 & 27,92 \\
\hline 1992-1998 & 154 & 7,41 & 35,34 \\
\hline 1993-1999 & 182 & 8,76 & 44,10 \\
\hline $1994-2000$ & 117 & 5,63 & 49,74 \\
\hline $1995-2001$ & 109 & 5,25 & 54,98 \\
\hline $1996-2002$ & 131 & 6,31 & 61,29 \\
\hline $1997-2003$ & 126 & 6,07 & 67,36 \\
\hline $1998-2004$ & 122 & 5,87 & 73,23 \\
\hline $1999-2005$ & 91 & 4,38 & 77,61 \\
\hline $2000-2006$ & 36 & 1.73 & 79,35 \\
\hline $2001-2007$ & 49 & 2.36 & 81,70 \\
\hline $2002-2008$ & 96 & 4,62 & 86,33 \\
\hline 2003-2009-I & 83 & 4,00 & 90,32 \\
\hline |-2005-|-2010 & 47 & 2,26 & 92,59 \\
\hline 2005-II-2010-II & 24 & 1,16 & 93,74 \\
\hline 2006-I-2011-I & 36 & 1,73 & 95,47 \\
\hline $2006-|I-2011-| \mid$ & 42 & 2,02 & 97,50 \\
\hline 2007-|-2012-I & 52 & 2,50 & 100,00 \\
\hline Total & 2077 & 100,00 & \\
\hline$\overline{\bar{X}}=86,5 ; \quad M_{e}=87 ; \quad M_{o}=36 ;$ & 158; Min & $x=182 P_{2}$ & $75=120,75$ \\
\hline
\end{tabular}

\section{TABLA 4}

\section{Modalidad de obtención de título universitario Facultad de Enfermería 1981-2012}

\begin{tabular}{|c|c|c|c|c|c|c|c|c|c|c|c|c|}
\hline \multirow{3}{*}{ Año academic } & \multicolumn{10}{|c|}{ Modalidad } & & \\
\hline & \multicolumn{2}{|c|}{$\begin{array}{c}\text { Complement } \\
\text { ación } \\
\text { académica }\end{array}$} & \multicolumn{2}{|c|}{$\begin{array}{l}\text { Actualizació } \\
\text { n académica }\end{array}$} & \multicolumn{2}{|c|}{$\begin{array}{l}\text { Proyecto de } \\
\text { inversión }\end{array}$} & \multicolumn{2}{|c|}{$\begin{array}{l}\text { Suficiencia } \\
\text { académica }\end{array}$} & \multicolumn{2}{|c|}{ Tesis } & \multicolumn{2}{|c|}{ TOTAL } \\
\hline & $\mathbf{f}_{\mathrm{i}}$ & $\mathbf{p}_{\mathrm{i}} \%$ & $\mathbf{f}_{\mathrm{i}}$ & $\mathbf{p}_{\mathrm{i}} \%$ & $\mathbf{f}_{\mathrm{i}}$ & $\mathbf{p}_{\mathrm{i}} \%$ & $\mathbf{f}_{\mathrm{i}}$ & $\mathbf{p}_{\mathrm{i}} \%$ & $\mathbf{f}_{\mathrm{i}}$ & $\mathbf{p}_{\mathrm{i}} \%$ & $\mathbf{f}_{\mathrm{i}}$ & $\mathbf{p}_{\mathrm{i}} \%$ \\
\hline $2000-2006$ & - & - & - & - & - & - & 36 & 100 & - & - & 36 & 100 \\
\hline $2001-2007$ & - & - & 22 & 44,90 & 8 & 16,33 & 1 & 2,04 & 18 & 36,73 & 49 & 100 \\
\hline $2002-2008$ & - & - & 24 & 25,00 & - & - & 44 & 45,83 & 28 & 29,17 & 96 & 100 \\
\hline ו-2003-2009 & - & - & 20 & 24,10 & 39 & 46,99 & 2 & 2,41 & 22 & 26,51 & 83 & 100 \\
\hline |2005-I-2010-I & - & - & 20 & 42,55 & - & - & 1 & 2,13 & 26 & 55,32 & 47 & 100 \\
\hline 2005-II-2010-II & - & - & - & - & - & - & - & - & 24 & 100,00 & 24 & 100 \\
\hline |-2006-|-2011 & 1 & 2.78 & - & - & - & - & - & - & 35 & 97,22 & 36 & 100 \\
\hline 2006-II-2011-II & - & - & - & - & - & - & - & - & 42 & 100,00 & 42 & 100 \\
\hline 2007-|-2012-I & 2 & 3.85 & - & - & - & - & - & - & 50 & 96,15 & 52 & 100 \\
\hline Total & 3 & 0,65 & 86 & 18,49 & 47 & 10,11 & 84 & 18,06 & 245 & 52,69 & 465 & 100 \\
\hline
\end{tabular}




\section{DISCUSIÓN.}

La Facultad de Enfermería tiene 25 años de Formación Académica, contando con las siguientes características:

\section{A. Entrada. Admisión: Número de Vacantes que oferta la Facultad de Enfermería en los 5 últimos años. (Cuadro} $\mathbf{N}^{\circ}$ 1). El $60 \%$ de los vacantes corresponde al rubro admisión ordinaria y el $40 \%$ al Centro Pre Universitario (CEPU). Demanda para ingreso/captación de alumnos: ratio postulantes- ingresantes. Alta demanda de ingreso. El ratio vacantes/ingresantes a la Facultad de Enfermería 2009l fue de 11,53, el 2009 II fue de 8,6\%.Lo que nos demuestra que este ratio es bueno. Cantidad de Postulantes por Ingresantes, 2010. El ratio general de Vacantes Ingresantes en las universidades públicas fue de $4,8 \%$ en las privadas de $1,2 \%$. Los 10 grupos de carreras profesionales de mayor demanda de alumnos de pre grado, 1996 y 2010 fue: Año 1996 la demanda de alumnos en las Facultades de Enfermería fue de $6,3 \%$ en el año 2010 fue de 6,8\% a nivel nacional (4). Selectividad de acceso a la universidad (15 $\%)$, definido por el número de candidatos (postulantes) que compiten por una vacante. A mayor cantidad de postulantes se asume una mayor calidad de estudiantes, debido a la competitividad para acceder a la plaza (5).

\section{B. Proceso.}

Docentes. Número de docentes. En la Facultad de Enfermería los últimos 11 años, no ha variado la plana docente contando con 58 docentes de ellos 09 docentes tienen el grado de Doctorado de ellos 08 son de la especialidad, en lo que respecta a la Maestría de los 21 docentes solo 06 son de la especialidad, en el caso de la Segunda Especialidad de los 22 docentes, solo 21 docentes son de la misma especialidad. Según Marcelo Rojas Cairampoma. Universidad Peruana: Estadísticas y referentes internacionales. 2007. Las Calificaciones académicas de los docentes
(10\%). Número de docentes con los grados de maestría y doctorados. Otro novedoso indicador, también de interés para nuestra realidad, ahora con el tema de la homologación docente, resulta conveniente que las universidades tengan una relación constantemente actualizada de los grados académicos de sus profesores (6).

Docentes. Por categoría y clase: Desde el año 1984 que contaron con 2 docentes, la frecuencia fue ascendente hasta el año 1990 llegando a 27 docentes hasta el año, y desde el año 2003 hasta la fecha se mantienen con 58 docentes. Se puede observar la distribución de los docentes jefes de práctica predominan desde los inicios hasta 1994, en la historia de la facultad de enfermería a la actualidad se puede rescatar la frecuencia de docentes auxiliares desde el año 1986 hasta el 1994, reapareciendo nuevamente en el año 2000 , estableciendo una variedad a partir del año 2002 con la frecuencia de docentes a tiempo completo, asociados y principales. El promedio de alumnos por asignatura para los semestres académicos 2009- II, 2010-I, 2010II y 2011-I es de 45 por aula. La relación Alumnos / docentes es de 9,34 alumnos por docente, para el semestre 2010-II. Teniendo 542 alumnos matriculados y 58 docentes. El promedio de estudiantes por profesor en prácticas de laboratorio es de 45/1 profesor. El promedio de estudiantes por profesor en la práctica clínica para el semestre 2010 I y 2010-2 es de 5,5 por profesor. Según la encuesta EL 69 \%de los alumnos refiere QUE SI está de acuerdo. Satisfechos con número de alumnos para las clases teóricas y prácticas. Cantidad de Alumnos de Pre-Grado por Docente Universitario. 2010. El ratio docente alumno en las Universidades Públicas es de $14,4 \%$.en las privadas $12,6 \%$ Tanto en el año1,995 como en el 2,000, las instituciones públicas se encuentran en condición favorable para una mejor selección de postulantes (7,4 y 8,6 postulantes por cada ingresante, respectivamente), a diferencia de las entidades privadas donde la posibilidad de selección se mantiene muy baja en ambos 
años (1,4 y 1,1 respectivamente). Incidencia en la calidad del producto, necesidad de incrementar difusión, reducir vacantes, implementar cursos de nivelación.

\section{Estudiantes: Promedio ponderado (Cuadro} $\mathbf{N}^{\circ}$ 05).- El promedio ponderado de notas de acuerdo al registro de la Facultad de enfermería se ajustan a una distribución normal con una media de 15,02, y una desviación estándar de 1,17. Se afirma que: El máximo promedio alcanzado fue de 17,78. El mínimo promedio aprobatorio alcanzado fue 11,04. El rendimiento estudiantil es una característica compleja cuya evaluación involucra un gran número variable, observable $o$ no. Se han realizado numerosas investigaciones acerca del rendimiento y se concluye que la nota, a la que el conglomerado universitario alude como signo del "rendimiento", es sólo una pequeña parte de esta definición. Existen diversas opiniones acerca de las innumerables causas que pueden afectar al rendimiento estudiantil, pero en esta investigación no se hace alusión a las causas sino que se propone el estudio de varias dimensiones para medir el rendimiento, estas son: la calidad, la cantidad, el abandono y la velocidad en los estudios. En este trabajo se propone una metodología, que la autora ya ha aplicado y comprobado en anteriores trabajos de asesoría, acerca del rendimiento estudiantil. El camino a seguir es: Análisis de Componentes Principales, cálculo de una ecuación matemática $y$, si existen grupos comparativos de estudiantes, un Análisis Unifactorial de Varianza (7).

Tipo de Institución educativa de los que proceden los alumnos: El $76 \%$ han estudiado en una institución educativa estatal y el $24 \%$ lo han realizado en una institución particular. Según el Censo Nacional Universitario 2010 (II CENAUN) muestra que la procedencia de los estudiantes de pre grado vienen en su mayoría de instituciones educativas estatales (62,8\%), seguido de las instituciones educativas particulares $(26,7 \%)$ y en menor cantidad de las instituciones educativas religiosas $(9,7 \%)$. Como es de esperarse la procedencia de estudiantes de instituciones educativas estatales es mayor en las universidades públicas $(76,2 \%)$, pero aún en las universidades privadas, la mayor parte de los estudiantes proceden de las instituciones educativas estatales (54,0\%). (8). El $90 \%$ son del sexo femenino y el $10 \%$ del sexo masculino. Según el Censo Nacional Universitario 2010 (II CENAUN) PERÚ: Alumnos de pre grado, por sexo, 2010 (9). El (51 $(51,1 \%)$ son de sexo masculino y el $(48,9 \%)$ son de sexo femenino (10). El promedio de edad de los alumnos de acuerdo al registro de la Facultad de enfermería se ajustan a una distribución normal con una media de 21 años, y una desviación estándar de 3,38 . El $51,1 \%$ de los alumnos tienen las edades comprendidas entre los 17 a 20 años, el $36,3 \%$ de 21 a 24 años, y el $7,1 \%$ de los alumnos tienen las edades comprendidas entre 25 a 28 años. Siendo la edad promedio de culminación de la secundaria a los 16 años, el ingreso a la universidad debería darse al año siguiente de egresar la secundaria, es decir, a los 17 años de edad. Según los resultados del ii censo universitario 2010 INEI - ANR (11). En la práctica son muy pocos los jóvenes que logran ingresar a un centro superior de estudios a esta edad. Así, la edad promedio de ingreso a la universidad, es de 18,9 años de edad, siendo 0,4 años mayor en hombres que en mujeres y 0,5 años de diferencia entre públicas y privadas (12). El currículo establecido a inicios del desempeño académico en el año 1981 hasta los 1999 (19 años) es de tipo tradicional. El año 2000 al 2001 currículo durante la Comisión Reorganizadora (CORE). Desde el año 2002 hasta la actualidad se viene desarrollando el currículo del tipo constructivista. W. Peñaloza, señala que "...tradicionalmente los currículos profesionales han sido cognoscitivos, y su estructura era simple: un listado de las diversas disciplinas científicas indispensables para adquirir los conocimientos atañederos a 
una profesión", las instituciones educativas han instilado la idea que "educar"= dar conocimientos, y es la manera como los docentes conciben su "misión". Del análisis de la situación postula el currículo integral compuesto por Áreas: de conocimiento (formación general y profesional,), de prácticas profesionales, de actividades no cognoscitivas, de orientación y consejería y de investigación (13). De acuerdo con la Teoría del Constructivismo, el Aprendizaje es una (re)construcción de conocimientos ya elaborados y el sujeto que aprende es un procesador activo de la información y el responsable último de dicho aprendizaje, con la participación del maestro como un facilitador y mediador del mismo y, los más importante, proveedor de toda la ayuda pedagógica que el alumno requiera. Los planes de estudio desde inicios de la facultad de enfermería fue diseñado anualmente hasta el año 2000, en el año 2001 además de ser anual se estableció la programación semestral y desde el año 2002 hasta la fecha se viene programando semestralmente, respaldada por resoluciones. Desde el año 1981 hasta la actualidad las horas promedio del plan de estudio respecto a las teorías es de 2427,9, siendo el menor número de horas de 1649 en el año 2000 y el máximo de 3281 en el año 1986-87. Respecto a las horas de prácticas, el promedio de horas es de 3135,4 , en el año 2003 el número de horas prácticas fue el menor de todos (1904) y el máximo número de horas prácticas fue en el año 1986 y 1987 con 6248 horas. Las horas de prácticas pre profesionales son en promedio 1143.1, siendo el mínimo 900 horas durante los años 1998 al 2000 y 2003 al 2005, y el máximo en el año 2002 con 1350 horas. El total de horas académicas es en promedio de 6607. El número de crédito mínimo establecido fue en el año académico 1993 con 198 créditos, y el máximo fue al año siguiente, en el 1994, con 231 créditos, establecido de igual manera en el año 2007. Siendo el promedio de créditos desde 1981 hasta la actualidad de 219,4. Según la
Agencia Nacional de Educación de la Calidad y Acreditación informa de la distribución del plan de Estudios, por tipo de materia y créditos de la Facultad de Enfermería de la Universidad de Granada de España contiene Formación básica 60 créditos, Obligatorias 78 créditos, Optativos: 12 créditos; Práctica externa 80 créditos; Trabajo fin de grado 10 créditos. Con un total de créditos de 240 créditos. El RD 1125/2003, de 5 de septiembre que regula el Sistema Europeo de Transferencia de Créditos (ECTS) dice que: "El crédito europeo es la unidad de medida del haber académico que representa la cantidad de trabajo del estudiante para cumplir los objetivos del programa de estudios y que se obtiene por la superación de cada una de las materias que integran los planes de estudios de las diversas enseñanzas conducentes a la obtención de títulos universitarios de carácter oficial y validez en todo el territorio nacional. En esta unidad de medida se integran las enseñanzas teóricas y prácticas, así como otras actividades académicas dirigidas, con inclusión de las horas de estudio y de trabajo que el estudiante debe realizar para alcanzar los objetivos formativos propios de cada una de las materias del correspondiente plan de estudios".

Con estas condiciones se presenta la propuesta de las horas de dedicación del alumno que recoge las actividades presenciales, que sin las prácticas clínicas, supondrán el $40 \%$ de las horas, entre las que tendremos: clases teóricas, talleres, seminarios, grupos de discusión, y otras actividades que incluirán al menos un $10 \%$ de las horas como tutorías, siendo el $60 \%$ restante horas no presenciales del alumno dedicadas al estudio y trabajo necesarios para alcanzar los resultados de aprendizaje de las competencias correspondientes a su perfil profesional. En las universidades de Europa el número de estudiantes es el siguiente: Seminarios: 10 a 15 alumnos, Prácticas simuladas/sala de demostración. 6 a 8 alumnos, Prácticas clínicas: 2 a 6 
alumnos. Estos serían a grandes rasgos aquellos aspectos específicos que son imprescindibles para la titulación (15).

\section{SALIDA.}

Se puede concluir que existen diferencias relevantes entre las preferencias de los diferentes tipos de modalidades de titulación, con un declive en la preferencia respecto a la suficiencia académica, actualización académica y proyecto de inversión, se observa además una estable preferencia por la complementación académica y un notable ascenso en la titulación por modalidad de tesis. En los últimos años son el $100 \%$ la obtención de título universitario por TESIS. La Comisión de Ciencia y Tecnología del Congreso analiza cinco iniciativas legislativas para fomentar la investigación en el Perú. Con el fin de impulsar la investigación en el país, la Comisión de Ciencia y Tecnología del Congreso analiza establecer la obligatoriedad de la presentación de la tesis para que los estudiantes universitarios obtengan su título profesional. Así lo adelantó el titular de dicho grupo de trabajo, Pedro Spadaro, quien alertó sobre los bajos índices de investigación. Para cambiar este panorama, dijo que se impulsan hasta cinco iniciativas legislativas. "Queremos modificar la ley universitaria para que el joven que está por titularse ya no opte por un examen para su grado, sino que opte por una tesis productiva y enfocada a resolver los problemas de índole regional", indicó a Andina. Asimismo, dijo que buscará dialogar con los rectores de las principales universidades del país para abordar el tema. Cabe recordar que la actual Ley Universitaria señala en su artículo 23 que para obtener el título de Licenciado "o sus equivalentes, se requiere la presentación de una tesis o de un examen profesional" (16).

\section{CONCLUSIONES}

Existen diferencias relevantes entre las preferencias de los diferentes tipos de modalidades de titulación, con un declive en la preferencia respecto a la suficiencia académica, actualización académica y proyecto de inversión, se observa además una estable preferencia por la complementación académica y un notable ascenso en la titulación por modalidad de tesis. En los últimos años son el $100 \%$ la obtención de título universitario por tesis.

\section{RECOMENDACIONES:}

Continuar con este tipo de trabajos de investigación, porque permite conocer la historia de la facultad y su contesto académico y socio político.

\section{Correspondencia:}

Heddy Sofía Manrique Manrique

Correo electrónico:

heddymanrique@hotmail.com

\section{REFERENCIAS BIBLIOGRAFICAS}

1. Ministerio de Comercio Exterior y Turismo: Ica. (Texto en internet). (Citado el 12 de junio del 2013). Disponible en: http://www.proinversion.gob.pe/Repositorio APS/0/0/JER/ICA ESTADISTICAS/sectores\%20economicos. xls]

2. Quintanilla M, et al. Curriculum Facultad de Enfermería Univ. Nac. San Luis Gonzaga de Ica. Ica, Perú. 2012.

3. Oficina de Personal .Universidad Nacional San Luis Gonzaga de Ica. 2009.

4. Roja, M. Universidad Peruana: Estadísticas y referentes internacionales. Universidad Mayor de San Marcos. Lima, Perú. 2007.

5. Garnica, E. El rendimiento estudiantil: una metodología para su medición. Instituto de Investigaciones Económicas y Sociales Universidad de Los Andes. Venezuela. 1997. (Texto en internet). (Citado el 12 de junio del 2013). Disponible en: http://iies.faces.ula.ve/Revi sta/Articulos/Revista 13/ Pdf/Rev13Garnica.pdf

6. Resultados del II Censo Universitario 2010 INEI - ANR. Lima, Perú. 2010. 
7. Sánchez A. II Censo Nacional Universitario. Lima, Perú. 2010. p 13.

8. Peñaloza, W. 1988. Tecnología educativa. Escuela empresarial andina del convenio Andrés Bello. 2da ed. Lima. p. 26.

9. Murillo Tapia, Manuel Abraham Armando Percepción de las enfermeras sobre el ejercicio de la profesión de enfermería en el Hospital Nacional Arzobispo Loayza.LIMA.PERU-2010

10. Germán Bes, Concepción Miqueo, Consuelo. Historia de la Institución de la Enfermería Universitaria: Análisis con perspectiva de género. Universidad de Zaragoza, España. 2006.
11. Miralles S. et,al. Purificación. Historia de la Escuela de Enfermeras "Salus Infirmorum» Madrid, España: 2008

12. Diario El Comercio. Proponen que la tesis sea único requisito para obtener título profesional. Lima, Perú. 30 de Septiembre del 2011.

Recibido: 13/02/14

Aprobado para publicación: 14/05/14 